\title{
Human disc cells in monolayer vs 3D culture: cell shape, division and matrix formation Helen E Gruber ${ }^{1}$ and Edward N Hanley Jr. ${ }^{1}$
}

Address: ${ }^{1}$ Department of Orthopaedic Surgery, Carolinas Medical Center, Charlotte, N.C.

E-mail: ${ }^{1}$ hgruber@carolinas.org ${ }^{1}$ ehanley@carolinas.org

\begin{abstract}
Background: The relationship between cell shape, proliferation, and extracellular matrix (ECM) production, important aspects of cell behavior, is examined in a little-studied cell type, the human annulus cell from the intervertebral disc, during monolayer vs three-dimensional (3D) culture.

Results: Three experimental studies showed that cells respond specifically to culture microenvironments by changes in cell shape, mitosis and ECM production: I) Cell passages showed extensive immunohistochemical evidence of Type I and II collagens only in 3D culture. Chondroitin sulfate and keratan sulfate were abundant in both monolayer and 3D cultures. 2) Cells showed significantly greater proliferation in monolayer in the presence of platelet-derived growth factor compared to cells in 3D. 3) Cells on Matrigel ${ }^{\mathrm{TM}}$-coated monolayer substrates became rounded and formed nodular colonies, a finding absent during monolayer growth.

Conclusions: The cell's in vivo interactions with the ECM can regulate shape, gene expression and other cell functions. The shape of the annulus cell changes markedly during life: the young, healthy disc contains spindle shaped cells and abundant collagen. With aging and degeneration, many cells assume a strikingly different appearance, become rounded and are surrounded by unusual accumulations of ECM products. In vitro manipulation of disc cells provides an experimental window for testing how disc cells from given individuals respond when they are grown in environments which direct cells to have either spindle- or rounded-shapes. In vitro assessment of the response of such cells to platelet-derived growth factor and to Matrigel ${ }^{\mathrm{TM}}$ showed a continued influence of cell shape even in the presence of a growth factor stimulus. These findings contribute new information to the important issue of the influence of cell shape on cell behavior.
\end{abstract}

\section{Background}

Information on the in vitro behavior of human intervertebral disc cells is still relatively scarce in spite of the large health care expenditures associated with degenerative disc disease. Chelberg et al have studied human disc cells cultured in alginate beads [1], and several other laboratories have recently studied disc cells from a variety of animals $[2,3,4,5,6,7,8,9]$. Although these cells are most easily established initially in monolayer culture, this microenvironment may not be permissive for many extra- cellular matrix (ECM) component which the cells produce in vivo.

The present report studies the relationship between cell shape, proliferation and ECM production by human cells cultured from the annulus. Previous work from our laboratory has reported culture methods for these cells, their proliferative response to TGF- $\beta$ and Matrigel ${ }^{\mathrm{TM}}$, and the anti-apoptotic effects of IGF-1 (insulin-like growth factor-1) and PDGF (platelet-derived growth factor) $[10,11,12]$. 3D culture results in a rounded cell phe- 
notype, increased proteoglycan synthesis compared to monolayer grown cells, and formation of multi-celled colonies with ECM deposited around and between cells $[11,13]$. In the present study, cells from sequential passages were seeded into either monolayer or $3 \mathrm{D}$ culture and assessed with immunohistochemistry for ECM products; cells were also evaluated in monolayer vs $3 \mathrm{D}$ culture to assess the mitogenic effects of PDGF, an antiapoptotic cytokine for annulus cells [10]. Cells were also grown on a plastic substrate coated with the reconstituted basement membrane extract Matrigel ${ }^{\mathrm{TM}}$, which contains multiple growth factors.

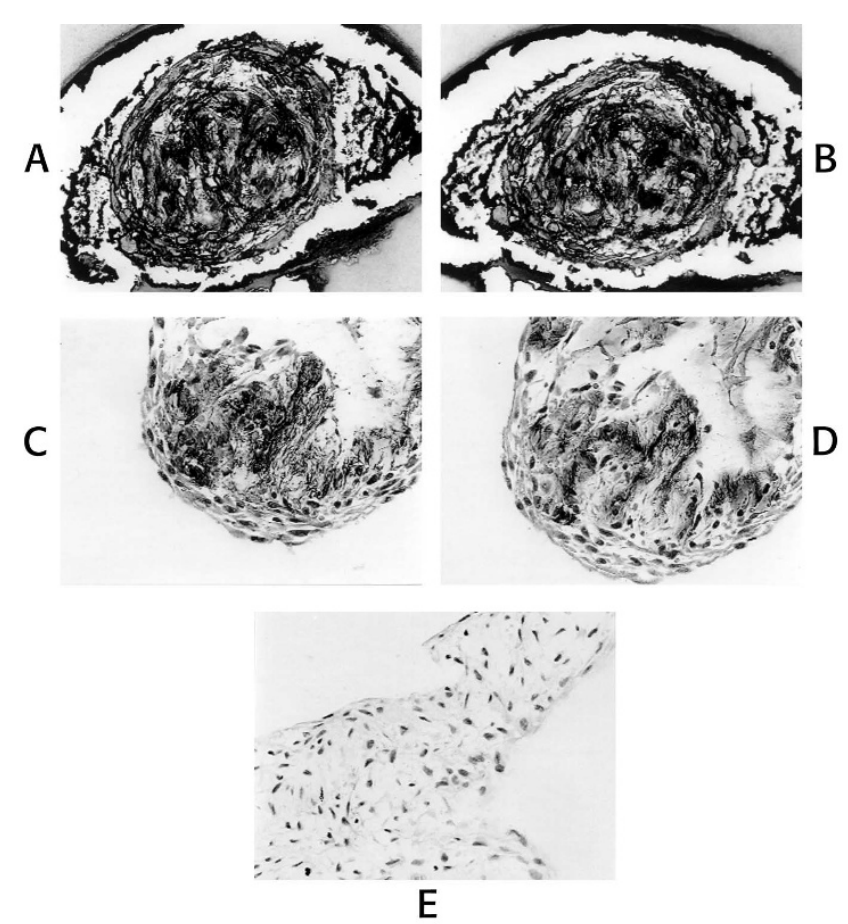

Figure I

Photomicrographs of ECM immunolocalizations of cells grown in the 3D microenvironment: Figures I and I are serial sections through a multi-celled colony evaluated for localization of chondroitin sulfate (Fig. I), keratan sulfate (Fig I). Figures $I$ and $I$ are serial sections through a multi-celled colony evaluated for localization of Type I collagen (Fig. I), and Type II collagen (Fig. I). Note positive black localization product for these ECM components. Figure I is a photomicrograph from a different specimen which was used as the negative control with deletion of primary antibody for this series of localizations with hematoxylin staining. (All photomicrographs $X$ 295). Cells were from the same subject, were first passage and were grown for 10 days.

\section{Results}

Monolayer vs 3D culture and ECM production:

Human cells from the annulus of four subjects were taken from passages 1 through 4 and seeded either into monolayer or $3 \mathrm{D}$ alginate microenvironments.
When grown in 3D culture, human disc cells form multicelled colonies (Figure 1). All four ECM components evaluated here (Type I and II collagen, chondroitin sulfate and keratan sulfate) were present in sectioned colonies of cells in $3 \mathrm{D}$ culture over the course of passages 1 through 4 (Figure 1111). Quantitative analysis shown in Table 1 presents the percentage of colonies which showed each of the ECM components at the various passage times. There were no statistically significant changes over the course of the four passages with respect to the $\%$ of colonies showing production of each ECM component.

When grown in monolayer culture, human disc cells assume a flattened, spindle-shaped morphology. The proteoglycans chondroitin sulfate and keratan sulfate showed abundant localizations between and around the cultured cells (Figure 2 and 2). Monolayer cultures, however, showed only very sparse, very rare, small localizations for either Type I or II collagen (Figure 2 and 2).
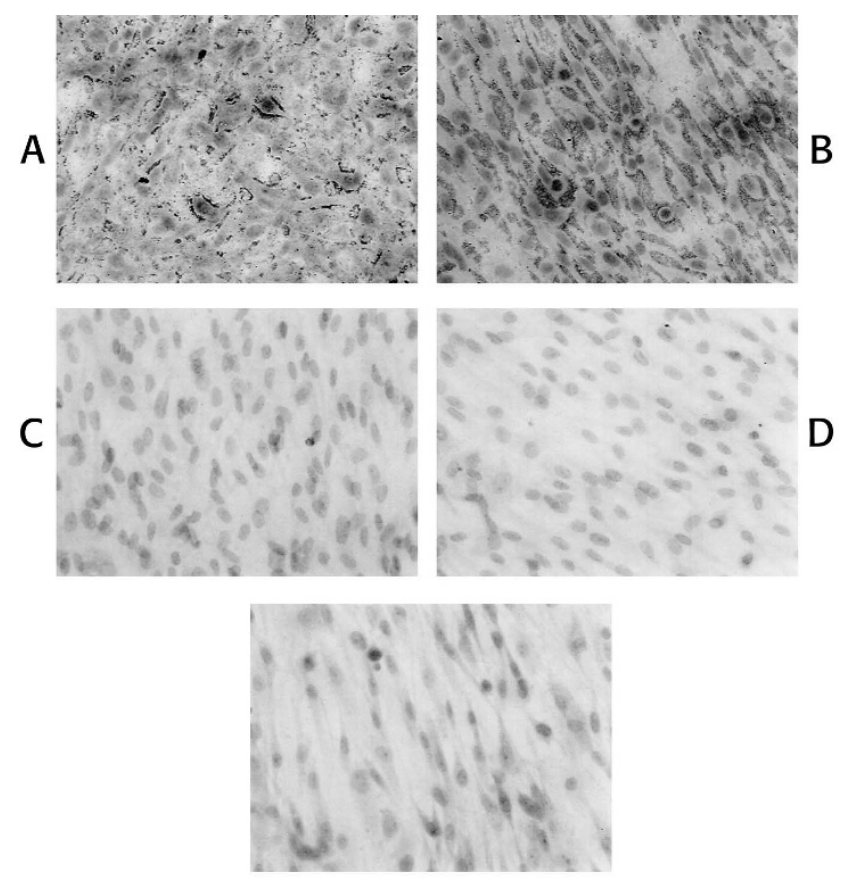

$E$

Figure 2

Photomicrographs of ECM localizations performed on whole cells grown in monolayer culture: For these studies, cells from the same subject were plated at the same density in 6 wells of a multi-well chamber slide and cultured for 10 days. Immunolocalizations were performed for each ECM product using a separate well. Prominent deposits of chondroitin sulfate (Fig. 2) and keratan sulfate (Fig. 2) can be seen surrounding the cells. In contrast, localizations for Type I collagen (Fig. 2) and Type II collagen (Fig. 2) show no deposition of these collagens by monolayer cultured cells. Figure 2 was taken from a well used as the negative control with deletion of primary antibody for these localizations with hematoxylin staining. (All photomicrographs, X 295). 


\section{Cell proliferation studies for monolayer vs 3D cultures:} Comparison of growth in MEM with 20\% FBS (the normal growth conditions for these cells) in monolayer vs $3 \mathrm{D}$ culture was carried out with cells grown under normal culture conditions and cells grown in the presence of PDGF. Under normal growth condition, monolayer culture produced a significantly greater $\left[{ }^{3} \mathrm{H}\right]$-thymidine uptake/ $\mu \mathrm{g}$ DNA than did 3D culture (mean $\left[{ }^{3} \mathrm{H}\right]$-thymidine uptake/ $\mu \mathrm{g}$ DNA for monolayer: 18,909 \pm 7,299 (mean \pm s.e.m.) vs $5,469 \pm 4048$ for $3 \mathrm{D}, \mathrm{p}=0.039$.

Our interest in the potential mitogenic effect of PDGF on annulus cells stems from our previous finding that 100 $\mathrm{ng} / \mathrm{ml}$ has can rescue or retard apoptosis in vitro [10]. In the present study, this dose was applied to cells and compared to control cells receiving only 1\% FBS (needed to maintain cell health). In monolayer culture, proliferation was significantly greater in monolayer cells (expressed as a \% of controls) $(990.7 \% \pm 261.1)$ compared to the more modest increase seen in $3 \mathrm{D}$ cultured cells $(435.8 \% \pm$ 150.0) $(\mathrm{p}=0.02)$.

\section{Culture on Matrigel ${ }^{\mathrm{TM}}$-coated surfaces:}

Annulus cells grown on Matrigel $^{\mathrm{TM}}$-coated monolayer surfaces displayed a rounded cell phenotype and with expansion during culture formed mounded nodular colonies (Figure 3). Cells grown on control uncoated wells displayed a flattened, spindle-shaped phenotype. Cells maintained this rounded configuration throughout 8 days of culture.

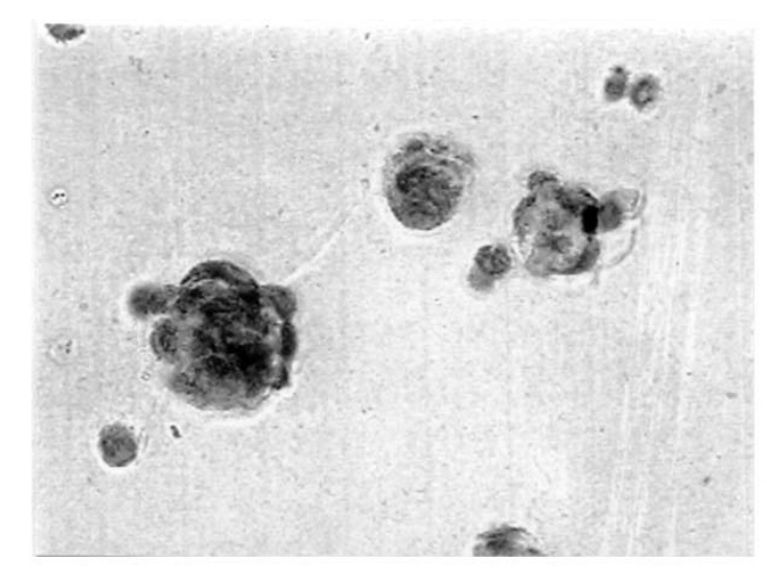

Figure 3

Photomicrograph of the rounded cell phenotype and mounded colonies formed by cells growing on Matrigel ${ }^{\mathrm{TM}}$-coated plastic. This is a markedly different cell phenotype than is seen with growth on uncoated plastic as shown in Figure 2 (X 295).
Quantitative Scoring of the \% of Colonies Containing Type I Collagen, Type II Collagen, Chondroitin Sulfate or Keratan Sulfate*

\begin{tabular}{lcccc}
\hline & $\begin{array}{c}\text { Type I Col- } \\
\text { lagen }\end{array}$ & $\begin{array}{c}\text { Type II Col- } \\
\text { lagen }\end{array}$ & $\begin{array}{c}\text { Chondroitin } \\
\text { Sulfate }\end{array}$ & $\begin{array}{c}\text { Keratan } \\
\text { Sulfate }\end{array}$ \\
& & & & \\
\hline & & & & \\
Passage 1 & $93.9 \pm 3.7$ & $86.2 \pm 1.7$ & $90.0 \pm 3.8$ & $80.9 \pm 7.0$ \\
Passage 2 & $96.7 \pm 1.0$ & $85.1 \pm 3.0$ & $87.3 \pm 2.1$ & $83.5 \pm 2.6$ \\
Passage 3 & $98.1 \pm 0.6$ & $88.5 \pm 2.3$ & $91.6 \pm 3.9$ & $85.1 \pm 3.0$ \\
Passage 4 & $97.4 \pm 0.5$ & $85.2 \pm 1.6$ & $96.2 \pm 1.1$ & $87.2 \pm 2.3$
\end{tabular}

*Data are mean \pm s.e.m. for colonies scored for the presence of the specific ECM component. Data are based on a mean of 240 colonies scored on each subject's cells cultured for each passage; cells from five individuals were evaluated.

\section{Discussion}

Results presented here are important since they expand our understanding of disc cell function in two markedly distinct tissue culture environments, monolayer and $3 \mathrm{D}$ culture, and focus upon four major ECM products important to disc biology. The matrix components of the healthy intervertebral disc supply both the tensile strength (derived from its collagen network) and shock absorber cushioning (derived from its proteoglycan content) which allow the disc to successfully meet the loading and motion needs of the spine. The present in vitro studies are also important since they afford an experimental opportunity to modulate cell shape, ECM production and proliferative capabilities in a human cell type about which there is still relatively little in vitro information. Although annulus cells have frequently been characterized as chondrocyte-like in the literature, these are a distinctive cell type about which much is still to be learned. We believe that this is the first study which documents differences in the response of disc cells from individuals cultured in either monolayer vs $3 \mathrm{D}$ conditions at respective passages. Our studies, however, involved a number of assumptions which are considered below and which have limited the interpretation of our findings.

Although quantitative data were obtained on the proportion of $3 \mathrm{D}$ colonies producing ECM components, ECM production in monolayer culture was evaluated only as "present" or "absent" since it was not possible to distinguish which adjacent cells laid down the ECM material. Future studies would be more informative if they included quantitative biochemical determination of ECM products or if levels of gene expression for the ECM products were determined. Such methods would eliminate the time-intensive serial sectioning of $3 \mathrm{D}$ colonies needed for more detailed histologic evaluation. Another issue of importance is the derivation of cells from different spinal levels; it should be borne in mind that cervical, thoracic 
and lumbar discs are subjected to different stress and strain conditions which may be reflected in the experimental behavior cells in vitro.

It should also be noted that although we have re-created environments in vitro which mimic cell shape features in the young and in the aging/degenerating disc, there are certainly a wide variety of factors which contribute to the function and proliferation of disc cells in vivo which were not included in our in vitro experiments. Such issues include factors associated with the decreased vascularity of the disc in the adult and alterations in stress patterns during aging and physical activity.

In spite of these limitations, the present study has provided insight into the proliferative and functional behavior of disc cells in vitro. Serial passage studies of annulus cells in monolayer and $3 \mathrm{D}$ culture showed that proteoglycan expression occurs in monolayer culture but that this microenvironment minimizes the cells production of collagen. It should be noted that the studies of monolayer cells did not evaluate ECM components that might have been secreted into the medium. Future studies are planned to assess ECM production using biochemical media assays; however, in some cultures small amounts of Types I and II collagen were detected, showing that matrix could have been detected using the immunohistochemical protocols used in the present work. In the $3 \mathrm{D}$ microenvironment, however, matrix is retained between and around the cells which produced it; this enhanced our ability to morphologically detect ECM products. Disc cells appear similar to chondrocytes in their monolayer and 3D ECM production capabilities; it has been shown by a number of studies that chondrocytes in monolayer appear to de-differentiate and that subsequent $3 \mathrm{D}$ culture leads to re-expression of ECM products characteristics of in vivo chondrocyte behavior $[17,18,19]$.

Glowacki et al have suggested that cell shape is an important factor in chondrocytes and designed studies in which shape was held constant by modification of the substrate adhesiveness with poly(2-hydroxyethyl methacrylate); cells held in the rounded phenotype proliferated slowly, incorporated low levels of thymidine into DNA, and incorporated large amounts of sulfate into glycosaminoglycans [20]. Chondrocytes retained in the fibroblast-like shape were flattened, showed more rapid growth, greater thymidine incorporation, and lower sulfate uptake. They concluded that cell shape played an important role in phenotypic expression. Other experiments by Folkman and Moscona using bovine aortic endothelial cells showed similar findings in that as cells were modified from the flatten to spheroid shape, cell proliferation decreased. Direct comparison of the proliferative ability of human disc cells is somewhat more difficult for both monolayer and $3 \mathrm{D}$ culture since disc cells are extremely slow growers, often requiring a month to establish even primary cultures in a confluent state in flasks. Studies here showed that cultures in monolayer exhibit a significantly greater proliferation than do the same cells seeded into $3 \mathrm{D}$ culture. Our studies here with PDGF show a similar response to this cytokine by disc cells: the \% increase in proliferation compared to controls was $990 \%$ in monolayer culture, but only $435 \%$ in the same passage and same cells seeded into the $3 \mathrm{D}$ culture environment.

PDGF was selected as a cytokine of interest for this work because of our previous demonstration of its anti-apoptotic effects on disc cells [10; 21]. PDGF has been shown in a number of studies to be a potent mitogen in vitro [22], and it also signals other cell responses such as survival [23] and transformation [24]. The effect of PDGF on disc cells grown in monolayer cells vs $3 \mathrm{D}$ growth appears to involve the degree of response: monolayer and $3 \mathrm{D}$ cells both showed a proliferative response to this cytokine; it is interesting that IGF-1 did not produce a mitogenic response at the doses at which it induces antiapoptotic rescue (data not shown).

Matrigel $^{\mathrm{TM}}$, a commercially available soluble basement membrane extract, contains multiple growth factors (o$0.1 \mathrm{pg} / \mathrm{ml}$ basic fibroblast growth factor; 0.5-1.3 ng/ml epidermal growth factor; $15.6 \mathrm{ng} / \mathrm{ml}$ IGF-1; $12 \mathrm{pg} / \mathrm{ml}$ PDGF, $<0.2 \mathrm{ng} / \mathrm{ml}$ neuronal growth factor, and $2.3 \mathrm{ng} /$ $\mathrm{ml} \mathrm{TGF}-\beta[12]$. We have previously described the growth of disc cell colonies when plated within the Matrigel ${ }^{\mathrm{TM}}$ matrix; inside this matrix cells assume a rounded shape as was seen in the present work in which cells were plated on top of the Matrigel ${ }^{\mathrm{TM}}$ monolayer coasted upon the plastic substrate.

The effect of growth factors such as PDGF, an anti-apoptotic agent for annulus cells [10], and Matrigel ${ }^{\mathrm{TM}}$ are a relatively new but important area of research. Modulation of cell function in vitro adds to our understanding of the relationship and interaction between disc cells and their surrounding matrix. The effect of growth microenvironment, growth factors and disc cell proliferation and function adds new insight into our understanding of these important, but little studied, cells.

\section{Materials and Methods}

Clinical Specimens: Studies were approved by the Institutional Review Board. Control specimens were obtained from the Cooperative Human Tissue Network and surgical specimens from patients undergoing surgical disc procedures. Cells used in the study of monolayer vs $3 \mathrm{D}$ ECM matrix production assessed with immunohistochemistry were derived from the lumbar annuli of a 67 year old female donor and from four patients (two 35 year old males, a 53 year old male, and a 76 year old fe- 
male). Cells used in the study of the mitogenic responsiveness to PDGF in monolayer or $3 \mathrm{D}$ culture were derived from 12 subjects, mean age 47 years $( \pm 13.9$ yrs (S.D.)); 7 were males, 5 were females. The disc sites were lumbar in 9 subjects and cervical in 3. Cells grown on Matrigel $^{\mathrm{TM}}$ were derived from two cervical and two lumbar discs from two donors and two surgical patients (mean age 43 years; 2 males and 2 females). For all subjects studied, the mean Thompson score for gross disc morphology was grade III [14].

Cell Culture Methods: Primary cultures were grown as previously described [1112]. Following primary culture, cells were subsequently passaged in either monolayer culture (seeded at 5,00o cells/well of a Lab-Tek ${ }^{\mathrm{R}}$ Chamber Slide ${ }^{\mathrm{TM}}$ (Nunc, Napierville, IL) or into 3D culture in 1.2\% Keltone LV alginate (Kelco, San Diego, CA) at a cell density of 50,000 cells seeded into the alginate which was then layered onto a Costar Transwell Clear Insert (Costar, Cambridge, MA). Cells were grown as previously described in sterile modified Minimal Essential Medium with Earle's salts, non-essential amino acids, penicillinstreptomycin and 20\% fetal bovine serum [11]. Passages 1-4 were studied in parallel for ECM produced after 10 days of culture.

Analysis of ECM Production with immunohistochemistry: After 10 days of culture, alginate inserts were fixed in $1 \%$ neutral buffered formalin for 5 minutes, dehydrated, embedded in paraffin and serially sectioned en face. Monolayer cultures were fixed with cold acetone for 1 minute and stored in the cold until immunolocalization was performed.

Immunohistochemical localizations utilized rabbit antihuman collagen Type I (1:10o dilution) or rabbit anti-human collagen Type II (1:150 dilution) (Biodesign International, Kennebunk, ME), monoclonal antiproteoglycan delta DI-4S (ICN, Costa Mesa, CA), and monoclonal anti-keratan sulfate (Seikagaku Corp., Tokyo, Japan) using previously described techniques [15]. Negative controls were processed minus primary antibodies and also minus secondary antibodies. Localization utilized $\mathrm{DAB}$ colorimetric visualization of $\mathrm{ECM}$ components.

Analysis of ECM localization products in 3D cultures was performed using computer-assisted quantitative histomorphometry with the OsteoMeasure software (OsteoMetrics, Inc., Atlanta, GA). An average of 240 colonies/ passage/subject were evaluated. Data are presented as mean \pm s.e.m.

Analysis of Mitogenic Response to PDGF: Cells were fed $0.5 \mathrm{ml}$ per well minimum essential medium with Earle's salts prepared as described above except that fetal bo- vine serum was supplemented at $1 \%$ (MEM1) to constitute the control culture conditions ( $1 \%$ serum is necessary for healthy cell maintenance. Platelet-derived growth factor (PDGF; Sigma-Aldrich, St. Louis, MO) was added to MEM1 to achieve a final concentration of 100 $\mathrm{ng} / \mathrm{ml}$ PDGF, a dose shown in prior work to have anti-apoptotic effects on disc cells [10]. For monolayer culture, cells were seed at a density of $16,000 \mathrm{cell} /$ well in a 48 well culture plate; for agarose $3 \mathrm{D}$ culture, cells were seeded at a density of 50,000 cells/well in a 24 well culture plate and prepared as previously described. Cells grew for 10 days. DNA synthesis was measured by pulse labeling cultures with $2 \mu \mathrm{Ci} / \mathrm{ml}$ of $\left[{ }^{3} \mathrm{H}\right]$-thymidine (Amersham) during the last 24 hours of culture; aliquots were taken and analyzed to determine total DNA content using a fluorometric procedure as previously described $[11,16]$. Results were expressed as cpm $\left[{ }^{3} \mathrm{H}\right]$-thymidine uptake/ $\mu \mathrm{g}$ DNA. Each cell cultures was assessed in 3-5 replicate samples and results averaged.

Culture on Matrigel $^{\mathrm{TM}}$ : Cells from four subjects were derived as described above, trypsinized and plated at a density of 5,000 cells/well onto Nunc 8-well slides coated with 75-100 $\mu \mathrm{l}$ Matrigel $^{\mathrm{TM}}$ (Becton Dickinson, Bedford, MA) diluted 1:1 with MEM with 20\% FBS. Control wells were seeded with the same density of cells on uncoated wells. Cells were allowed to grow for 8 days, fixed with $1 \%$ neutral buffered formalin, rinsed with $70 \%$ ethanol, stained with toluidine blue, coverslipped and evaluated with light microscopy.

Statistical analysis: Statistical analysis of the 3D immunohistochemistry ECM data utilized SAS software (version 6.11, SAS Institute Inc., Cary, NC). A repeated measures analysis of variance was performed to test for differences over time; all tests were two-sided and p-values $<0.05$ were considered statistically significant. Data are expressed as mean \pm s.e.m.; paired t-tests were used in analyses of cell proliferation..

\section{Acknowledgements}

The authors thank Kelly Leslie, Shirley Coleman and Audrey Stasky for expert technical assistance. These studies were supported in part by the North American Spine Society and the Charlotte-Mecklenburg Health Services Foundation.

\section{References}

I. Chelberg MK, Banks GM, Geiger DF, Oegema TR Jr: Identification of heterogeneous cell populations in normal human intervertebral disc. J. Anat. 1955, 186:43-53

2. Poiraudeau S, Monteiro I, Anract P, Blanchard O, Revel M, Corvol MT: Phenotypic characteristics of rabbit intervertebral disc cells - Comparison with cartilage cells from the same animals. Spine 1999, 24:837-844

3. Chiba K, Andersson GBJ, Masuda K, Thonar EJMA: Metabolism of the extracellular matrix formed by intervertebral disc cells cultured in alginate. Spine 1997, 22:2885-2893

4. Chiba K, Andersson GBJ, Masuda K, Momohara S, Williams JM, Thonar EJMA: A new culture system to study the metabolism of the intervertebral disc in vitro. Spine 1998, 23:1821-1827 
5. Osada R, Ohshima $H$, Ishihara $H$, Yudoh K, Sakai K, Matsui $H$, Tsuji $\mathrm{H}$ : Autocrine/paracrine mechanism of insulin-like growth factor-I secretion, and the effect of insulin-like growth factor-I on proteoglycan synthesis in bovine intervertebral discs. J. Orthop. Res. 1996, 14:690-699

6. Ichimura K, Tsuji H, Matsui H, Makiyama N: Cell culture of the intervertebral disc of rats: Factors influencing culture, proteoglycan, collagen, and deoxyribonucleic acid synthesis. J. Spinal Disorders 1991, 4:428-436

7. Yoo JU, Papy RS, Malemud CJ: Suppression of proteoglycan synthesis in chondrocyte cultures derived from canine intervertebral disc. Spine 1992, 17:221-224

8. Maldonado BA, Oegema TR Jr: Initial characterization of the metabolism of intervertebral disc cells encapsulated in microspheres. J. Orthopaedic Res. 1992, 10:677-690

9. Thompson JP, Oegema TR Jr, Bradford DS: Stimulation of mature canine intervertebral disc by growth factors. Spine |99|, 16:253-260

10. Gruber HE, Hanley EN Jr: Anti-apoptotic effects of IGF-I and PDGF on human intervertebral disc cells in vitro. Spine 2000, 25:2। 53-2I57

II. Gruber HE, Fisher EC Jr, Desai B, Stasky AA, Hoelscher G, Hanley EN: Human intervertebral disc cells from the annulus: Threedimensional culture in agarose or alginate and responsiveness to TGF- $\beta$ I. Exp. Cell Res. 1997, 235: I3-2।

12. Desai BJ, Gruber HE, Hanley EN Jr: The influence of Matrigel ${ }^{\mathrm{TM}}$ or growth factor reduced Matrigel ${ }^{\mathrm{TM}}$ on human intervertebral disc cell growth and proliferation. Histol. Histopathol. 1999, 14:359-368

13. Gruber HE, Stasky AA, Hanley EN Jr: Characterization and phenotypic stability of human disc cells in vitro. Matrix Biol. 1997, 16:285-288

14. Thompson JP, Pearce RH, Schechter MT, Adams ME, Tsang IKY, BishOP PB: Preliminary evaluation of a scheme for grading the gross morphology of the human intervertebral disc. Spine 1990, 15:411-415

15. Schwob JE, Szumowski KEM, Stasky AA: Olfactory sensory neurons are trophically dependent on the olfactory bulb for their prolonged survival. J Neurosci. 1992, 12:3896-3919

16. Ramp WK, Dillaman RM, Lenz LG, Gay DM, Roer RD, Ballard TA: A serum substitute promotes osteoblast-like phenotypic expression in cultured cells from chick calvariae. Bone and Mineral 1991, I 5:1-17

17. Benya PD, Shaffer JD: Dedifferentiated chondrocytes reexpress the differentiated collagen phenotype when cultured in agarose gels. Cell 1982, 30:215-224

18. Benya PD, Padilla SR, Nimni ME: Independent regulation of collagen types by chondrocytes during the loss of differentiated function in culture. Cell 1978, I5:1313-1321

19. Von der Mark K, Gauss V, von der Mark H, Müller P: Relationship between cell shape and type of collagen synthesized as chondrocytes lose their cartilage phenotype in culture. $\mathrm{Na}$ ture 1977, 267:53I-532

20. Glowacki J, Trepman E, Folkman J: Cell shape and phenotypic expression in chondrocytes. Proc. Soc. Exp. Biol. Med. 1983, 172:9398

21. Desai BJ, Gruber HE: Anti-apoptotic actions of cytokines in mammalian cells. Proc. Soc. Exp. Biol. Med. 1999, 221:1-13

22. Deuel TF: Polypeptide growth factors: Roles in normal and abnormal cell growth. Ann. Rev. Cell Biol. 1996, 3:443-492

23. Barres BA, Hart IK, Coles HSR, Burne VF, Voyvodic JR, Richardson WS, Raff MC: Cell death and control of cell survival in the oligodendrocyte lineage. Cell 1992, 70:31-46

24. Deuel TF, Huang JS, Huang SS, Stroobant P, Waterfield MD: Expression of a platelet-derived growth factor-like protein in simian sarcoma virus transformed cells. Science 1983, 22 I: | 348-1350

\section{Pre-publication history}

The pre-publication history for this papercan be accessed here:

http://www.biomedcentral.com/content/backmatter/1471-2474-1-1-b1.pdf 